öGUM-Mitteilungen

Österreichische Gesellschaft für Ultraschall

\title{
in der Medizin
}

\section{O̊GUM-WEBINARE}

Als Vorteil und Chance der COVID-19-Pandemie kann die Beschleunigung der Digitalisierung angesehen werden, die unser berufliches und privates Leben verändern hat. Wir als ÖGUM möchten diese Gelegenheit nutzen und Webinare für unsere ÖGUM-Mitglieder anbieten. Diese ÖGUM-Webinare werden in monatlichen Abständen, jeweils mittwochabends, angeboten und sollen als aktuelle kurze Fortbildungen, Fallbesprechungen sowie Literaturübersichten erstellt werden. Wir freuen uns sehr, dass wir mit diesem Format nun die Möglichkeit haben, aktuelle Informationen zu transportieren, regelmäßigen Kontakt untereinander zu pflegen und damit auch unsere UltraschallCommunity zu stärken.

Die Webinare werden im Oktober 2020 starten:

7.10.2020 Alexander Spiel: Lungenultraschall in Zeiten der COVID-Pandemie
11.11.2020 Hannes Gruber: Ultraschallgesteuerte Behandlung des Karpaltunnelsyndroms

Im Dezember 2020 wird ein Webinar über ein pränatal-diagnostisches Thema (Philipp Klaritsch) und im Jänner 2021 über ein gynäkologisches Thema (Samir Helmy) angeboten werden.

Die Anmeldung dazu wird über die ÖGUMWebsite erfolgen. 